\title{
Damping trapped modes in an in-vacuum undulator at a synchrotron radiation light source
}

\author{
Kai Tian," James J. Sebek, Andrew D. Ringwall, and Zenghai Li \\ SLAC National Accelerator Laboratory, 2575 Sand Hill Rd, Menlo Park, California 94025, USA
}

(Received 14 February 2019; published 14 May 2019; corrected 5 June 2019)

\begin{abstract}
In-vacuum undulators have been widely operated in many synchrotron radiation facilities across the world. They usually are required to be operated at a smaller magnet gap than those of other undulators. Thus, operating challenges including impedance effects on the stored electron beam are introduced by these devices. In this paper, we report the efforts in solving the problem of coupled-bunch instabilities caused by an in-vacuum undulator in the SPEAR3 storage ring. Using beam based measurements, cold rf measurements, and numerical simulations, the source of the beam instabilities is characterized as trapped modes in the vacuum chamber. Using numerical models, we explored several approaches to reduce the strength of the trapped modes and found that ferrite dampers were the most effective and simplest way for mode damping in our SPEAR3 in-vacuum undulator. The results of the first rf cold measurement on an invacuum undulator equipped with these ferrite dampers agree well with numerical simulations.
\end{abstract}

DOI: 10.1103/PhysRevAccelBeams.22.050702

\section{INTRODUCTION}

Since the first in-vacuum undulator (IVU) was designed and operated in KEK [1] in the early 1990s, IVUs have been installed in various synchrotron radiation (SR) light sources around the world in order to expand the radiation spectrum to higher energies without the need to increase the beam energy [2-8]. In an IVU, the permanent magnet rows are inside a vacuum chamber, so the magnet gap is free from physical limitations and can be designed to be as small as possible. In order to obtain the higher harmonics of synchrotron radiation with a short undulator wavelength, a strong magnetic field is required. Therefore, a small magnet gap is often required for operating an IVU. In order to make the $x$-ray energy from the IVU tunable, the gap size must be adjustable. The beam generates strong electromagnetic fields in these small gaps. These fields act back on the beam and, depending on the physical design of the IVU chamber, may be sufficiently strong to drive a beam instability. The interaction strength of these fields and the beam is characterized by the impedance of the chamber.

Though there are some variants of structure design, most of these variants adopt some standard approaches to reduce the impedance effects on the electron beam passing through the gap $[9,10]$. For example, a modern IVU typically has

\footnotetext{
*ktian@slac.stanford.edu

Published by the American Physical Society under the terms of the Creative Commons Attribution 4.0 International license. Further distribution of this work must maintain attribution to the author(s) and the published article's title, journal citation, and DOI.
}

thin copper sheets which cover the magnet arrays to minimize the resistive wall impedance of the IVU as well as to shield the electron beam from the discontinuities resulting from the magnet poles. In addition, the flexible transitions [11] from the magnet pole pieces to the adjacent vacuum chambers have been carefully designed to provide a smooth tapered boundary along the beam path for all magnet gaps. Much work has been devoted to understand and calculate the impedance effects due to the IVUs [1214]. With decades of operational experience and numerous iterations of design efforts, the IVU is considered a mature technology in the community of SR light sources. However, most of the past studies on the IVU induced beam impedance effects have focused on the single bunch effects caused by short range wakefields. Until recently, long range wakefields caused by trapped rf modes in the IVU structures had been considered to be less important. Recently, various laboratories have reported their observations of coupled-bunch instabilities introduced by the IVUs [15-18]. In SPEAR3, we have also found similar beam instabilities associated with the addition of a recent IVU (BL15 ID). These observations demonstrate the importance of understanding the narrow band impedance of an IVU device.

SPEAR3 is a $3 \mathrm{GeV}, 500 \mathrm{~mA}$, third generation light source with a rf frequency of $476.315 \mathrm{MHz}$. The 2-meterlong BL15 insertion device in SPEAR3 is the second IVU in the storage ring with an undulator period of $22 \mathrm{~mm}$. The minimum operational magnet gap is designed to be $6.82 \mathrm{~mm}$. During early commissioning of the BL15 ID, we observed that the transverse beam size increased at small, discrete magnet gaps with $500 \mathrm{~mA}$ stored beam in 
the ring. Upon further investigation, we discovered that the beam size increase was caused by transverse coupledbunch instabilities driven by the trapped resonant modes inside the IVU chamber. The geometry of the IVU chamber, with the magnetic structure, resembles many features of a round ridge waveguide [19-21], which supports lower frequency modes that can be trapped inside the bare vacuum chamber. A transverse mode can be excited by the bunches in the ring. This mode, in turn, if sufficiently strong, can drive the beam unstable. The IVUs are designed for variable gap operations, therefore, the spectrum of the trapped modes shifts in frequency with the changes in the magnet gap of the IVU. The coupling impedance is sufficiently strong that when the mode frequency overlaps the lower vertical betatron sideband of a revolution harmonic [22], vertical instabilities are excited in SPEAR3. Most modern storage rings, including SPEAR3, are equipped with multibunch feedback systems, which have been successfully demonstrated to be effective in damping these, and other, transverse coupled-bunch instabilities [23-25]. However it is preferable to passively eliminate the source of the instability rather than to rely on active damping. In this paper, we will present a passive damping technique using ferrite dampers inside the IVU chamber to damp the trapped modes.

One direct way to reduce the interaction between the beam and the trapped rf mode is to decrease the transverse coupling impedance of the mode. If a structure is designed such that the mode frequencies exceed the cutoff frequency of the adjacent beam pipe, the fields in the mode will propagate out of the structure and the resonance will not build up to cause an instability. Otherwise, the transverse coupling impedance of a trapped mode is given by the following formula:

$$
Z_{\perp}=k(R / Q)_{\perp} Q_{L},
$$

where $k=\omega / c$ is the wave number of the mode, $(R / Q)_{\perp}$ is the transverse $(R / Q)$, and $Q_{L}$ is the loaded quality factor. The definition of $(R / Q)_{\perp}=V(r)^{2} /\left(\omega U k^{2} r^{2}\right)$ is chosen in the equation, wherein $V(r)$ represents the transit-time corrected voltage along the structure at a nonzero transverse offset of $r$. Equation (1) implies some approaches that can possibly work to damp trapped rf modes. First, the coupling impedance can be reduced by decreasing $(R / Q)_{\perp}$, which is the geometric factor of the resonant structure. Second, without changes to the geometry of the resonant structure, $Q_{L}$ can be damped using various types of $\mathrm{rf}$ dampers.

In the past, passive methods have been used to damp problematic rf modes caused by the ridge waveguide structure for the storage ring. These rf modes, found earlier in some beam chambers in the Advanced Photon Source (APS) due to the small gap between the beam chamber and the antechamber [26], corrupted the output from the beam position monitors. APS solved these problems using coaxial loop couplers and lossy ceramic loads at the narrow gap where the electric fields of the rf modes are mostly concentrated. However, in the SPEAR3 IVU chamber, it is impractical to apply any damping materials inside the magnet gap, both because of the movement of the gap and the need for any damping materials to stay far from the beam or the SR fan it generates. After thorough numerical simulations and $\mathrm{rf}$ measurements, we have designed ferrite based dampers and added them to the IVU structure, well away from the beam or x-ray trajectory, with minimum alteration of the original mechanical design of the device. These dampers have been integrated into the most recent IVU installed in SPEAR3, BL17 ID, which is identical to BL15 ID in terms of its structural design. Results from the cold rf, without electron beam, measurements for the BL17 ID chamber indicate that the dampers perform as designed.

To our knowledge, this is the first case of damping rf modes inside an ID chamber with lossy materials. Our study will be beneficial to the SR community in understanding the trapped rf modes inside an IVU chamber and preventing possible beam instabilities caused by them. The results we present in this paper can pave a path for future IVU designs that are passively stable with respect to coupled-bunch instabilities. The paper is structured as follows. Section II describes the characterization of the trapped modes using beam-based measurements, rf measurements, and numerical simulations. In Sec. III, we describe our setup for the electromagnetic simulations used to design the ferrite dampers. Section IV describes the mechanical design of the ferrite dampers. In Sec. V, we briefly discuss our study of possible alternate solutions to damp the trapped modes in an IVU chamber.

\section{CHARACTERIZATION OF TRAPPED MODES}

In order to passively damp the trapped mode inside the BL15 ID chamber, we first needed to understand the properties of the trapped modes. There are several ways to probe the rf modes in the chamber: beam based measurement to characterize the driving terms for the coupledbunch instabilities; direct (cold) $S$ parameter measurements of the trapped modes without electron beam; and numerical simulations to determine the rf properties of the trapped modes. In the following, we will present the results from all three of these approaches to confirm that we understand the characteristics of the trapped modes in the chamber.

\section{A. Beam based measurements}

The bunch-by-bunch feedback system in SPEAR3 not only allows us to effectively suppress the transverse beam instabilities induced by the BL15 ID but also enables us to carry out grow/damp measurements to characterize the impedances driving the beam instabilities. The grow/damp technique $[27,28]$ operates the machine above the instability threshold with feedback controlling the instability. 
By programming the feedback loop to be open for a short period of time, one allows the transverse beam motion to grow. At the end of that time, the feedback is turned back on and the beam motion is once again damped. The data for the bunch-by-bunch beam motion are then captured for modal analysis in order to extract the growth and damping rates of the individual coupled-bunch modes. During the measurement, in order to achieve a uniform filling pattern, the easiest case to analyze analytically, we fill all 372 buckets available in SPEAR3 to its operational $500 \mathrm{~mA}$ beam current. Due to the low ring impedance, the beam in SPEAR3 can still be passively stable with zero chromaticity at full beam current. Therefore we were able to conduct the measurement with a more linear optics by decreasing the vertical chromaticity of the storage ring to zero from its normal operating value of 2. Again, the reason to do so is for the ease of analytical analysis in the future.

The BL15 ID magnet gap is then scanned from its minimum value of $6.82 \mathrm{~mm}$ to $7.6 \mathrm{~mm}$ with a step size of $10 \mu \mathrm{m}$. Ion instabilities, SPEAR3's only coupled bunch instabilities prior to the addition of BL15 ID, are very weak, have slow growth rates, and are independent of gap settings. By contrast, higher order modes instabilities caused by the trapped rf modes in the IVU chamber are only excited over certain bands of gap values but have fast growth rates. From the grow/damp data at these gap values, the growth rates can be obtained from exponential fits of the amplitude growth of the dominant mode at each gap setting. Within the range of the magnet gap scan of the IVU, two modes sufficiently strong to create instabilities were found. Their growth rates are shown in Fig. 1, along with numerical fitting to the data points. Although the measurements were made with slightly different stored beam currents at each data point, we scaled all results to the values corresponding to a stored beam current of $500 \mathrm{~mA}$. The cutoff frequency of a ridge waveguide increases with the gap height. Therefore, when increasing the gap of the IVU, the frequency of the trapped mode will change continuously. In our case the change is sufficiently large that the ridge waveguide resonance can cross the lower sidebands of several adjacent revolution harmonics. With about a $0.8 \mathrm{~mm}$ change of gap, the stronger resonance, shown as series 1 in Fig. 1, travels through three coupled bunch instability modes: mode 156 through 158 . The circle markers represent measured data for mode 118, which is driven by another, weaker, ridge waveguide resonance. By fitting the measured data, shown as the dashed lines in Fig. 1, with Lorentzian functions, we can derive the gap values at the peaks and the quality factor of each resonance peak. Table I lists the results of the calculations.

From beam based measurements alone, we cannot determine the actual frequency of the trapped mode. One can only determine it modulo an integer number of harmonics of the SPEAR3 rf frequency. But, the results of rf measurements and numerical simulations all suggest

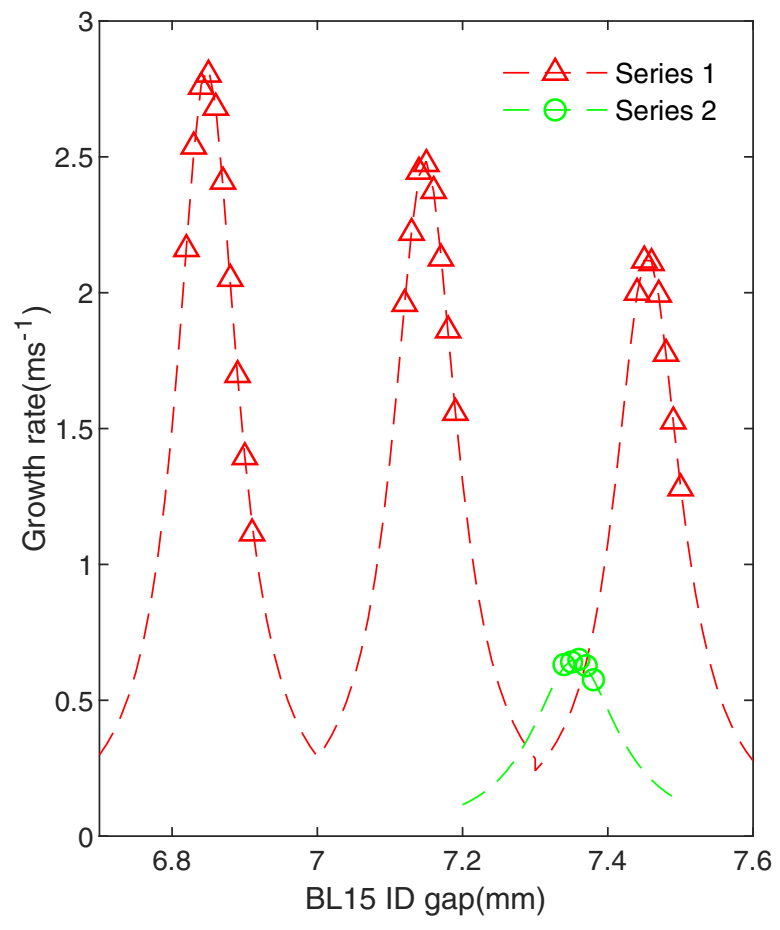

FIG. 1. Vertical coupled bunch instability growth rates in the IVU chamber.

that the trapped modes are at baseband. In other words, the mode frequency of the coupled bunch instability coincides with the resonant frequency of the trapped rf mode at the gap value for the peaks in Fig. 1. One should note that, in order to estimate the property of the trapped rf mode, we neglect the effects from damping terms to the transverse beam motion. As a reference, the vertical natural damping time in SPEAR3 is $5.3 \mathrm{~ms}$.

\section{B. $S$ parameter measurements}

Besides the beam based measurements, we have conducted $S$ parameter measurements of the BL15 ID chamber with an Agilent 8753ES network analyzer during an accelerator downtime. We installed two loop antennas into the chamber, so that we could perform $S_{21}$ measurements. One antenna is at the center port of the ID chamber and the other one is at an upstream port about $64.8 \mathrm{~cm}$ away. Both antennas are identical; each has a $94 \mathrm{~mm}$ diameter loop

TABLE I. Mode properties derived from beam based measurements.

\begin{tabular}{lccr}
\hline \hline Gap $(\mathrm{mm})$ & Mode Index & Mode Freq.(MHz) & $Q$ \\
\hline 6.85 & 156 & 199.51 & 456 \\
7.15 & 157 & 200.86 & 444 \\
7.45 & 158 & 202.12 & 446 \\
7.36 & 118 & 150.84 & 237 \\
\hline \hline
\end{tabular}




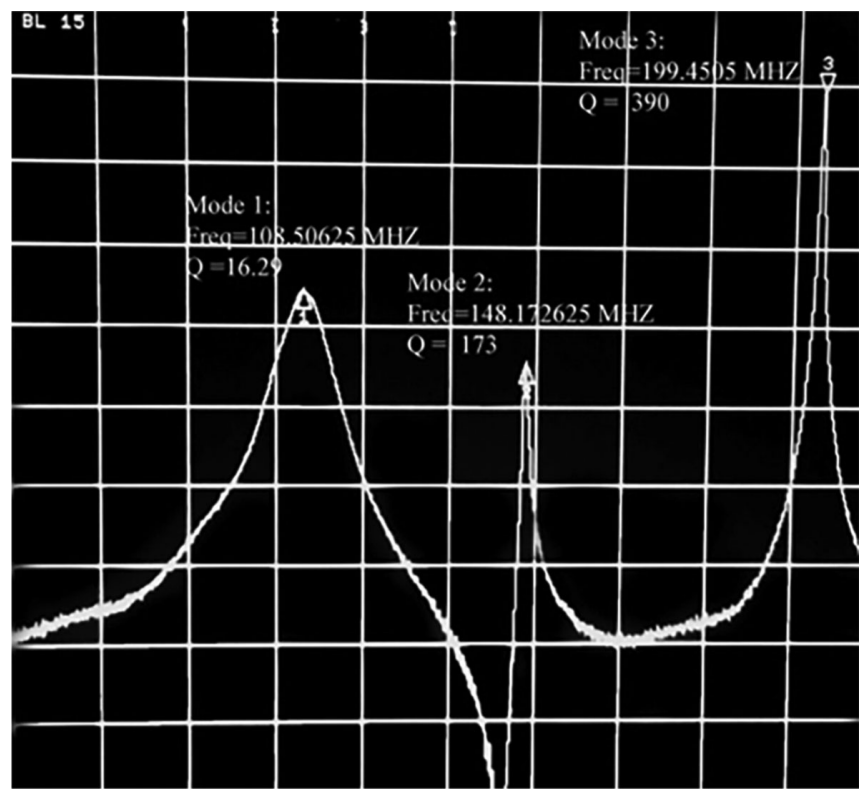

FIG. 2. Screenshot of the $S$ parameter measurement on the IVU chamber.

connected to an N-type feed-through. The loop size was maximized to maximize the coupling to the high order modes (HOMs) modes in the chamber. However, for an rf mode frequency of about $200 \mathrm{MHz}$, the calculated free space radiation impedance from the antenna is only about $0.3 \Omega$, so we would expect relatively weak coupling for the base-band modes in this measurement.

During the measurement, we first set the BL15 ID gap to $6.85 \mathrm{~mm}$ and scanned the network analyzer from $100 \mathrm{MHz}$ to around $1 \mathrm{GHz}$; the measurement result is shown in Fig. 2. Only three peaks significantly are above the noise levels; they are resolved at $108.51 \mathrm{MHz}$ (Mode \#1), 148.17 MHz (Mode \#2), and 199.45 MHz (Mode \#3). Recalling the data in Table I, we estimate the resonant frequency for the trapped mode introducing instabilities should be $199.51 \mathrm{MHz}$ at a $6.85 \mathrm{~mm}$ gap. Extrapolation of the data in Table I yields that the resonant frequency, at $6.82 \mathrm{~mm}$ gap, should be about $199.38 \mathrm{MHz}$, which is $0.04 \%$ from the measured resonant frequency of mode \#3. In addition, the third mode has a $Q$ of 390, the highest $Q$ of these three modes. Based on these facts, we can conclude that mode \#3 is the trapped rf mode that drive the series 1 coupled-bunch instability in Fig. 1. This can be further verified by varying the gap of BL15 ID to track the change of the resonant frequency of the trapped modes.

In Fig. 3, the black circular markers depict the measured resonant frequency of HOM \#3 at various gap settings of the IVU. Figure 3 shows the data fit both to a straight line (blue dashed line) and to a quadratic curve (red line); the quadratic function fits the data much better. Slater's theorem $[21,29,30]$ gives a differential expression for the change in frequency with respect to cavity shapes.

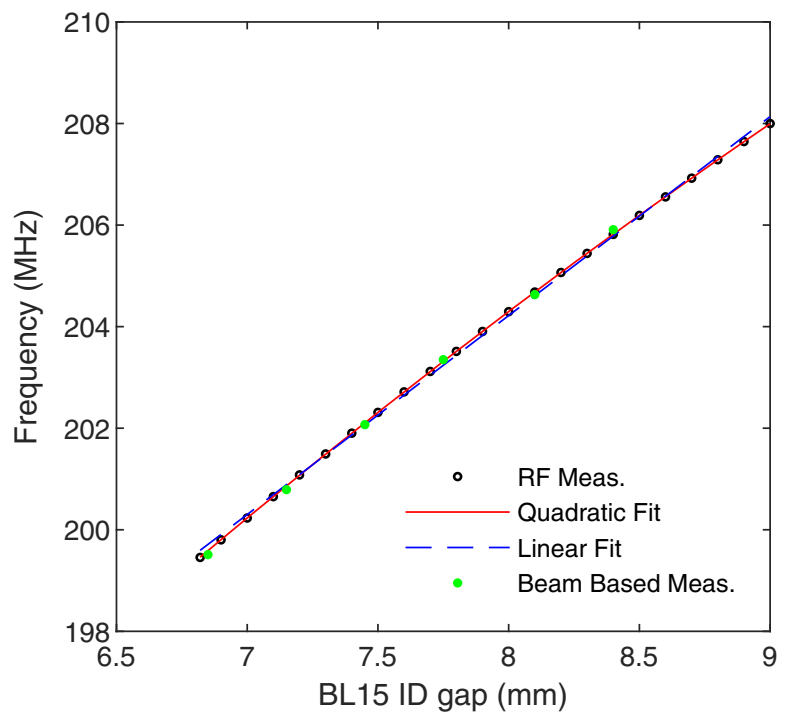

FIG. 3. Tracking the resonant frequency of the HOM \#3 at different BL15 ID gap.

$$
\delta \omega=\frac{-j \iint_{\Delta S} \mathbf{E}_{a}^{*} \times \mathbf{H} \cdot d \mathbf{S}}{\iiint_{V}\left(\epsilon \mathbf{E} \cdot \mathbf{E}_{a}^{*}+\mu \mathbf{H} \cdot \mathbf{H}_{a}^{*}\right) d v}
$$

where $\mathbf{E}_{a}$ and $\mathbf{H}_{a}$ are the fields in the unperturbed structure and $\mathbf{E}$ and $\mathbf{H}$ are the fields after the shape perturbation. An infinitesimal change of the gap will result in a linear change in the mode frequency. With each successive displacement of the undulator jaws, the definition of the unperturbed structure changes; we expect the linear coefficient that gives $\delta \omega$ in Eq. (2) to also change. In our case, the volume change of the IVU chamber, when varying the gap by about $2 \mathrm{~mm}$, is sufficiently large to show second order effects. This explains why we see the quadratic curvature in the fit of the resonant frequency of HOM \#3.

On the other hand, with beam based measurement, we can determine the ID gap settings when each coupled bunch instability mode is driven at the maximum strength. The frequency of each instability mode and its corresponding ID gap are illustrated by the green dots. It appears that the instability mode frequencies of all 6 modes (series 1) that can be observed in SPEAR3, are equal to the resonant frequency of HOM \#3 at the same BL15 ID gap. This is sufficient to prove that $\mathrm{HOM} \# 3$ is the source of the series 1 beam instability. Similarly, we can also conclude that series 2 instabilities are caused by HOM \#2.

\section{Numerical simulations}

To confirm the measured results, the IVU chamber is modeled numerically and solved using Omega3P [31] for TE modes up to $6 \mathrm{GHz}$ for over 1800 modes in total. (The simulation setup will be discussed thoroughly in the next section.) Here, we primarily focus on the results of the transverse coupling impedance and the unloaded quality factor $Q$ of these modes as shown in Fig. 4(a) and 4(b) 
(a)

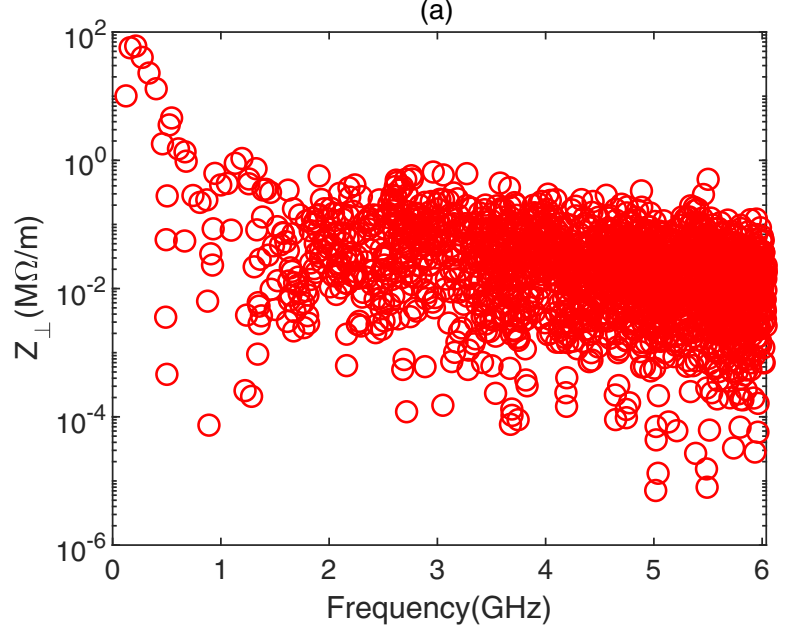

(b)

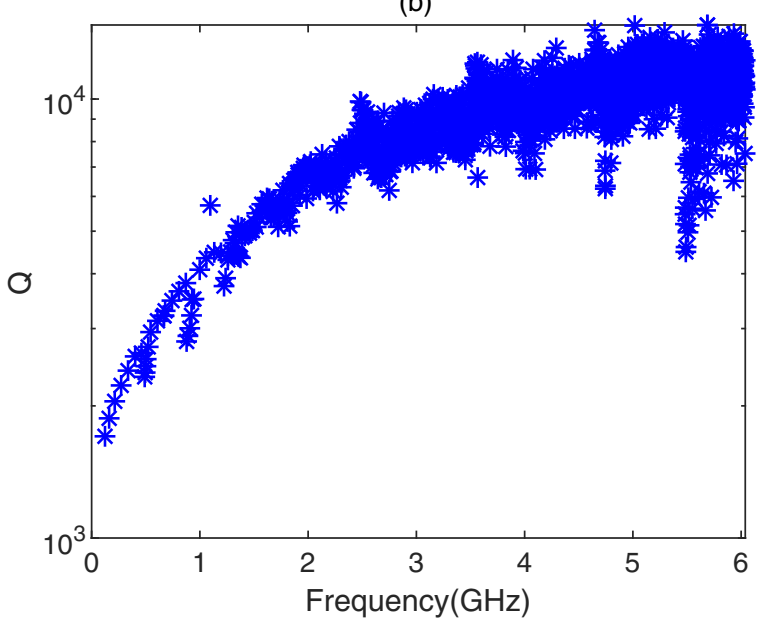

FIG. 4. Simulation results for TE modes up to $6 \mathrm{GHz}$.

respectively. There are two things worth noting in the simulation results. First, the transverse impedance of the second and third modes have higher impedances than the rest of the modes. Second, for the modes with resonant frequency higher than $1 \mathrm{GHz}$, the impedances drop by nearly two orders of magnitude. Both are consistent with our beam based and rf measurements and indicate that the base-band HOM modes cause the instabilities.

In Table II, we compare the resonant frequencies and quality factors of the first three TE modes obtained from simulations and $\mathrm{rf}$ measurements. The differences in the resonant frequencies for these three modes are on the order

TABLE II. Simulation results for the first three HOM modes.

\begin{tabular}{lcccc}
\hline \hline HOM\# & $f_{\text {sim }}(\mathrm{MHz})$ & $Q_{0}$ & $f_{\text {meas }}(\mathrm{MHz})$ & $Q_{L}$ (meas) \\
\hline 1 & 123.5 & 1705 & 108.5 & 16 \\
2 & 161.7 & 1873 & 148.17 & 173 \\
3 & 213.4 & 2048 & 199.45 & 390 \\
\hline \hline
\end{tabular}

of $10 \%$; however, the quality factors differ significantly. The quality factor calculated in the simulation is the intrinsic quality factor, or unloaded $Q$, which is determined by the surface power loss, while the measured quality factor is the loaded $Q$, which accounts for the total power loss including the coupling effect from the measurement instruments. Surface power loss in a resonant structure depends on many factors such as surface materials and roughness and can be very accurately calculated for $\mathrm{rf}$ cavities. However, in the IVU chamber, the large number of components and complex structures create unique challenges for the accurate modeling of the surface loss. Efforts have been spent to model the IVU chamber in more detail; the results show that with extra details in the structure and more careful attention to the surface materials, the unloaded $Q$ can be reduced by about a factor of 2 . We believe that the moderate improvement of the accuracy for the $Q$ calculation is insufficient to justify both the difficulties of adding these details into the numerical simulations routines and the increased computational time to do so.

\section{SIMULATION}

\section{A. Simulation model}

Numerical simulations for studying the HOM modes that cause the beam instabilities have been carried out using a parallel code, OMEGA3P, running on the super computer, Cori, at the National Energy Research Scientific Computing Center (NERSC) [32]. The simulation model mesh was constructed using CUBIT [33]; the data was visualized using ParaView [34]. The IVU chamber contains numerous components for various purposes. Therefore, it is necessary to build a model that is sufficiently simple to perform numerical simulations, while still good enough for accurately studying the $\mathrm{rf}$ properties of the structure. As discussed earlier, the mode frequency of interest for the IVU chamber is around $200 \mathrm{MHz}$. Therefore, as a rule of thumb, geometrical features with dimensions less than $10 \mathrm{~cm}$, or $1 / 15$ of the rf wavelength can be considered to be small and generally have less impact on the rf properties of the mode. We started with a highly simplified structure before gradually adding more features and keeping only those that significantly affect the simulation results.

The actual magnet row is $1954 \mathrm{~mm}$ long with transitions of lengths greater than $100 \mathrm{~mm}$ at each end. As shown in Fig. 5, the top and bottom magnet rows are attached to two I-beam shaped in-vacuum girders made of aluminum. Each of the two in-vacuum girders is connected to the out-ofvacuum mechanical gap motion system through 12 bellows link rods. These rods, which connect the girders and the outside chamber, have significant impacts on field patterns and frequencies of the rf modes. Therefore, we model the magnet row assembly with a $1954 \mathrm{~mm}$ long metal bar with a rectangular cross section measuring about the same size 


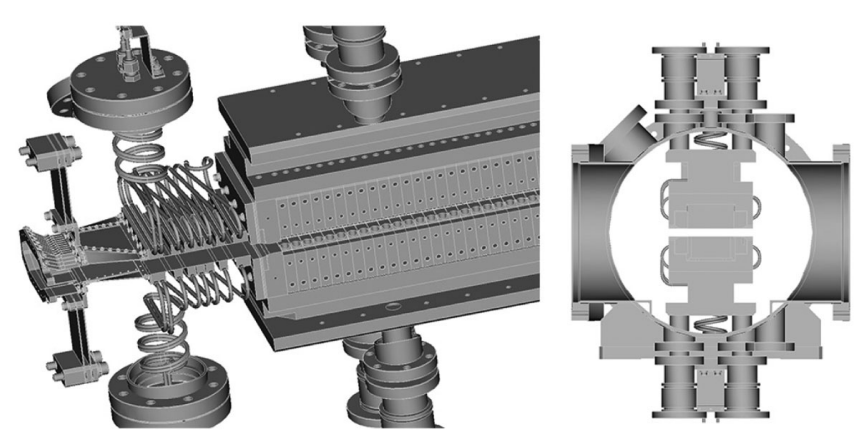

FIG. 5. The CAD model for the IVU chamber. Left: the IVU structure inside the chamber with the flexible transition; right: view of the cross section of the IVU device that resembles a round ridge wave guide. (Courtesy of Neomax Engineering Co.)

as that of the assembly. To reduce the resistive wall beam impedance and that contributed from the short range wakefields, a nickel-plated copper foil is attached to the permanent magnet (PMs) poles through magnetic attractive force to provide a highly conductive smooth surface on top of the magnet arrays. In addition, a flexible transition based on an SLS design [11] connects the PM gap to the entrance/ exit of the vacuum chamber. Due to thermal concerns, the transition is cooled by a complex water cooling system. Because the wavelengths of the modes of interest are much larger than the dimension of the cooling structure, a combination of a brick and a cylinder is used to represent the cooling system. The flexible section provides a smooth transition, but with the variation of the magnet gap, the profile for the transition is varied as well. Therefore, it is hard to model the exact boundary geometry for the electron beam when passing through the transition. Therefore we model the transition by first selecting several points along the transition, which fix the geometry relations to the magnet gap, and then linearly interpolate between them. As shown later, the transitions are critical to the transverse impedance for the trapped rf modes. Therefore, we believe the uncertainty of the model for the transition could significantly affect the simulation results. Finally, after considering the rf impacts of most of the major geometric features in the IVU chamber, we build for numerical studies a model as shown in Fig. 6.

\section{B. Damping schemes}

Although the IVU chamber supports numerous rf modes, as shown in Fig. 4(a), only those at lower frequencies have strong transverse coupling impedances and therefore a strong interaction with the electron beam. A close examination of the electromagnetic fields shows that the first six rf modes all belong to the same series of TE type resonant modes, each with a different longitudinal mode number. In the following, we will simply refer to them as the ridge waveguide modes. The first ridge waveguide mode, or $\mathrm{HOM} \# 1$, is the first harmonic with nodes only at the

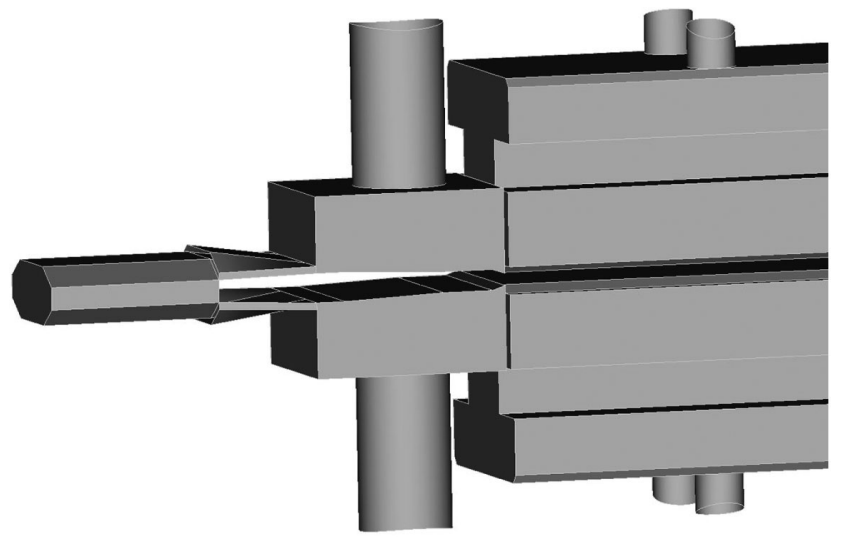

FIG. 6. The simplified numerical simulation model with the vacuum chamber being hidden.

longitudinal boundaries, giving a half cycle of $\mathrm{rf}$ field variation in the longitudinal direction. HOMs \#2 and \#3 are the second and the third harmonic with a full cycle and one and one-half cycles of field variation in the longitudinal direction, respectively.

In Fig. 7, we illustrate the distributions of electromagnetic fields of the ridge-waveguide modes in the IVU chamber using HOM \#3 as an example. Knowledge of these distributions is necessary in order to determine the type and location of electromagnetic absorbers that will be effective in damping the HOM fields. In the top image, the magnitude of the electric field on the inner surfaces of the device is color coded; arrows indicate the field direction. In the middle plot, the magnetic field on the outer surface of the chamber is similarly displayed. Finally, the color coded magnitude of the magnetic field on the inner surface of the IVU device is shown in the bottom image.
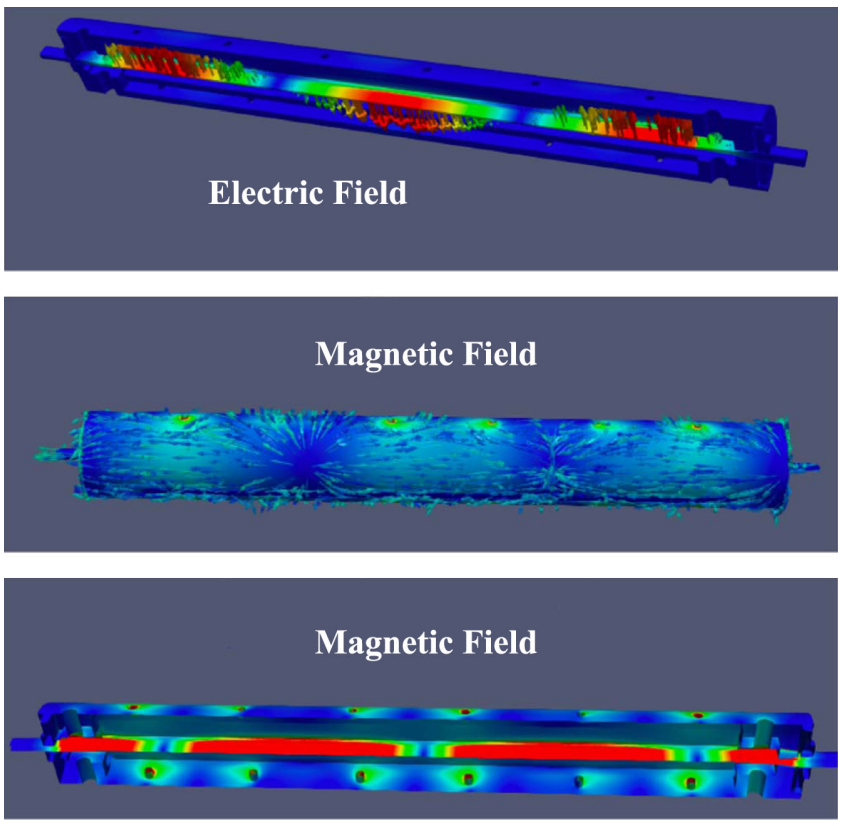

FIG. 7. Electromagnetic fields distribution for HOM \#3. 


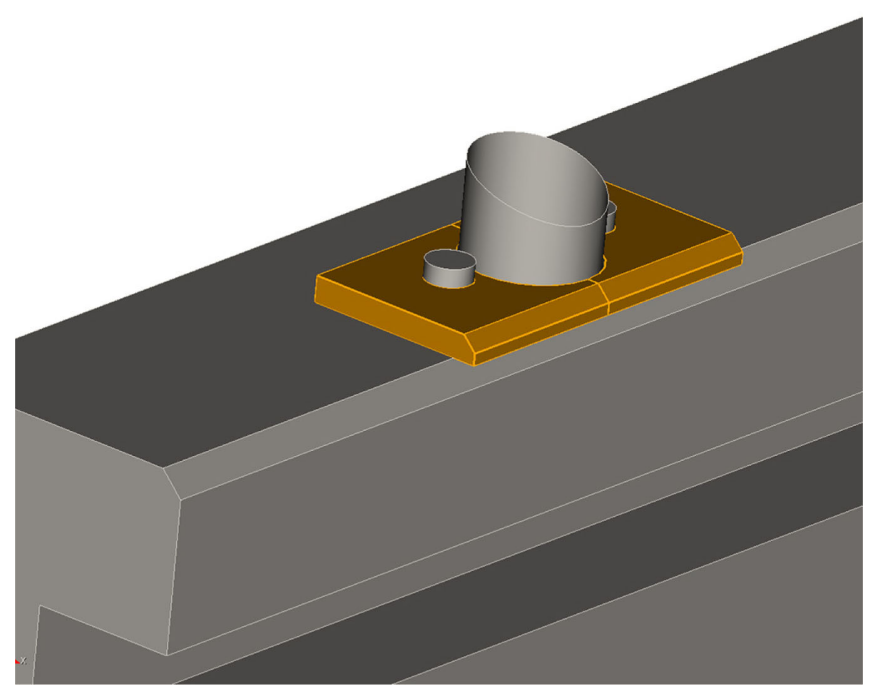

FIG. 8. The numerical simulation model for a pair of ferrite dampers.

From these plots, one can conclude that both the electric and magnetic fields of a ridge-waveguide mode are concentrated at the narrow magnet gap. Damping of both electric and magnetic fields is electromagnetically possible by placing the appropriate absorptive material in or near the gap. While the electric field in the rest of the chamber remain very small, significant magnet fields leak out of the magnet gap and fill the vast space in the chamber. Therefore, if one is limited by practical considerations to placing damping materials only away from the gap, we are limited to absorptive magnetic material. Especially, one should note that the magnetic field is enhanced around the bellows of the linked rods. Therefore, for HOM \#3, the mode can be effectively damped by adding magnetic damping materials around these rods. Furthermore, in order to achieve the full spectrum damping to all the ridge-waveguide modes, it is prudent to add the damping materials to all the rods.

The final design of the dampers consists of 24 pairs of ferrite brackets with each pair attached around each rod. Each ferrite is mounted on the girder using a stainless steel bolt with sufficient pressure to ensure tight contact between the ferrite and the girder for efficient thermal conductance. The simulation model for a pair of the ferrite bracket is shown in Fig. 8, where the bolts are also included in the model. For the damping materials, we have chosen commercially available ferrites, TT2-111, which have been experimentally tested for damping the HOMs in LHC collimators [35-37]. The detailed mechanical design of the ferrite dampers will be discussed in Sec. IV.

\section{Simulation results}

The permittivity and permeability of ferrite materials are both complex, frequency dependent quantities that can be represented by $\mu_{r}=\mu^{\prime}-i \mu^{\prime \prime}$ and $\epsilon_{r}=\epsilon^{\prime}-i \epsilon^{\prime \prime}$. Because OMEGA3P only allows fixed material properties for each

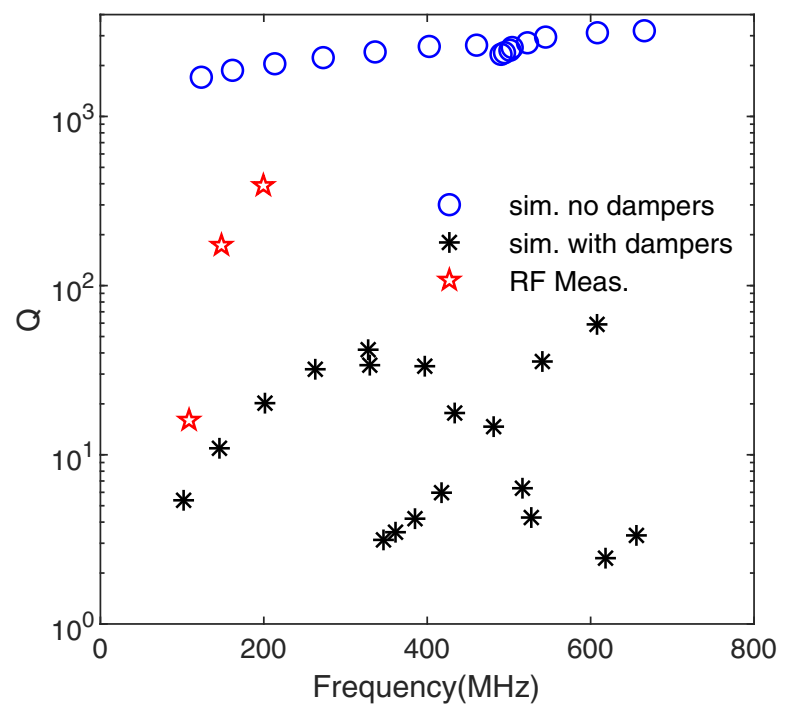

FIG. 9. Simulation results of quality factors of the trapped $\mathrm{rf}$ modes in the IVU with and without ferrite dampers.

run, we split the simulation into 4 runs to solve for the rf modes in different ranges of frequencies and change the material properties in each run so that we can take into account the frequency dependent variation of the ferrite material [37].

In Fig. 9, we plot the simulation results of the quality factors of the trapped rf modes up to $800 \mathrm{MHz}$ for the IVU chamber before (blue circles) and after (black stars) adding the ferrite dampers. For comparison, the $Q$ values of the three trapped modes measured in the cold rf measurements are also shown in the same plot (red pentagrams). As expected, after installing these ferrite dampers, the $Q$ values for all modes drop significantly. Particularly, for the first three modes, the loaded $Q$ drops by over two order of magnitude in simulations. With the dampers, the loaded $Q$ of HOM mode \#2 and \#3 would have comparable values with the measured $Q_{L}$ of HOM mode \#1 from the rf cold test. These indicate that both modes would be sufficiently damped such that they would not cause any beam instabilities. Besides providing significant damping effects, the ferrite dampers will slightly shift the resonant frequencies of the rf modes in the IVU chamber. The imaginary part of the permeability, $\mu^{\prime \prime}$, characterizes the capability of microwave power absorption for the damping material. Thus, it is relevant to study the dependence of the simulation results on the variation of $\mu^{\prime \prime}$. We repeat the simulation by varying the values of $\mu^{\prime \prime}$ by $\pm 10 \%$ from the nominal values and show the results in Fig. 10. These results indicate that the damping effects are not sensitive to small variations of the properties of the damping material. Another subject of concern during the design of the ferrite dampers is their power consumption and their resultant increase in temperature. One should note that while the beam is stable and stays on axis, the rf modes inside the chamber will have 


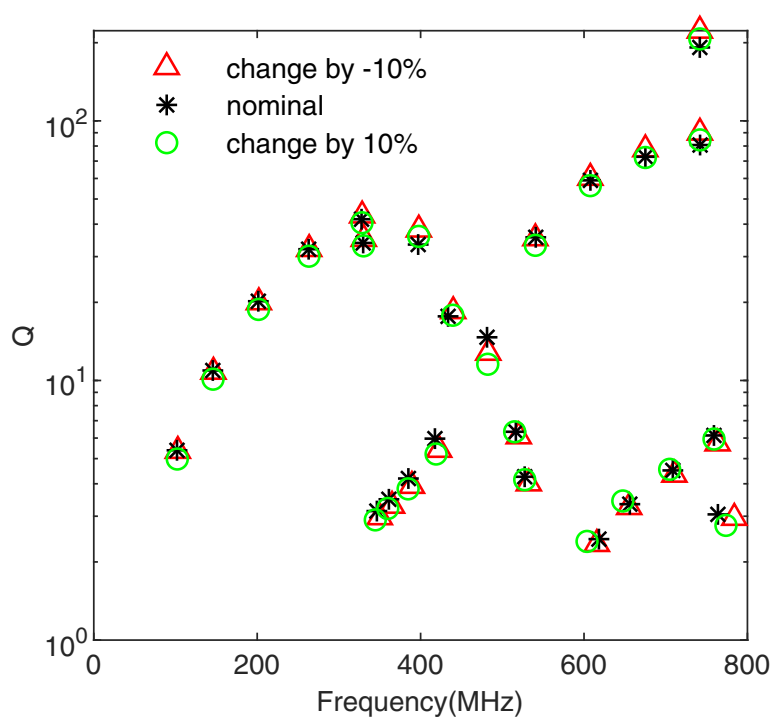

FIG. 10. Simlations results with varying ferrite materials properties.

little interaction with the bunch. Beam will lose power to the TE modes only when it oscillates transversely and couples to and excites the modes. For worst case scenarios, we assume the beam is deflected off-axis by $1 \mathrm{~mm}$ and calculate that the beam loses less than $35 \mathrm{~W}$ to all TE modes up to $2 \mathrm{GHz}$.

\section{MECHANICAL DESIGN}

The design and implementation of the ferrite damper pieces should accommodate the IVU design and operation with minimal modifications. Also the ferrite should not adversely affect the vacuum quality or IVU magnetic field.In this section, we will present the detailed mechanical design of the damper including ANSYS simulation results for the magnetic field interaction with the undulator poles and the thermal analysis.

As discussed, the material is a $\mathrm{NiZnFe}_{2} \mathrm{O}_{4}$ type ferrite, TT2-111R from Trans-Tech Inc. The TT2-111R ferrite has a thermal conductivity of $3.5 \mathrm{~W} / \mathrm{m}-\mathrm{K}$ and thermal expansion coefficient of $1.1 \mathrm{e}-5 / \mathrm{K}$. A sample of the ferrite measured by the SLAC vacuum group, using a precision vacuum conductance aperture, after $120^{\circ} \mathrm{C}$ bake, gives a room-temperature out-gassing rate of $6.5 \mathrm{e}-10 \mathrm{~T}-1 / \mathrm{s}-\mathrm{cm}^{2}$ [38]. For a similar ferrite used for the HOM absorbers on the KEK B-factory, T. Tajima et al. report a postbake, room-temperature outgassing rate of $5.0 \mathrm{e}-11 \mathrm{~T}-1 / \mathrm{s}-\mathrm{cm}^{2}$ with this rate rising to $2.0 \mathrm{e}-9 \mathrm{~T}-1 / \mathrm{s}-\mathrm{cm}^{2}$ at a temperature of $245^{\circ} \mathrm{C}$ [39]. Using the SLAC outgassing rate, the 48 ferrite pieces add $8.0 \mathrm{e}-7 \mathrm{~T}-1 / \mathrm{s}$ gas load to the IVU, so a fourth SPEAR-style titanium sublimation pump is added to an available port on the IVU chamber to compensate for this additional gas load. Even though the dissipated power in each ferrite piece is expected to be low (less than $4 \mathrm{~W}$ ),

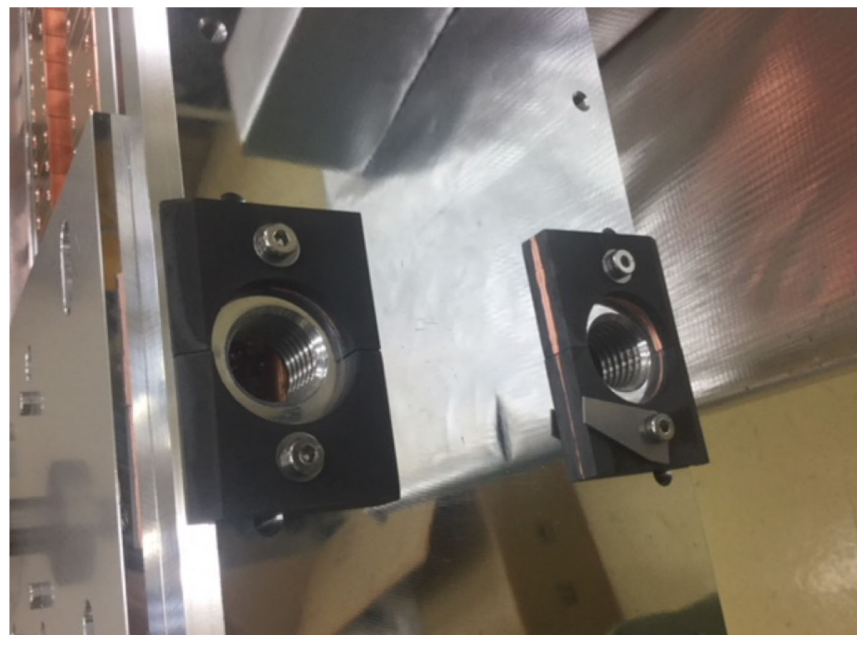

FIG. 11. Two pairs of ferrite dampers installed on top of the in vacuum girder of the SPEAR3 BL17 ID. (Courtesy of Neomax Engineering Co.)

the design requires sufficient heat transfer to limit the temperature rise and prevent high outgassing rates. Fortunately, the water-cooled aluminum beams supporting the IVU magnet arrays have an existing tapped hole pattern on either side of the linked rods available for fastening the 24 pairs of ferrite pieces. As shown in Fig. 11, the ferrite pieces are made from stock, $5 \mathrm{~mm}$ thick TT2-111R material. The ferrite pairs consists of a right handed and left handed piece, each $30 \times 40 \times 5 \mathrm{~mm}$, with a half circle relief to permit assembly around the rod with a $25-50 \mu \mathrm{m}$ gap left between the pieces to provide for thermal expansion. The M4 screws fasten each piece to the beam through a $5 \mathrm{~mm}$ diameter hole with a Bellville-style spring washer limiting the screw clamp force to $1000 \mathrm{~N}$ in order to avoid cracking the ferrite. Additionally, each piece has a chamfer so that the IVU magnet gap can be opened to $26 \mathrm{~mm}$ during magnet array assembly with clearance maintained between the ferrite pieces and the IVU vacuum chamber. Copper plating on the ferrite contact surface that mates to the watercooled aluminum beam is used to improve the ferrite heat transfer and limit the temperature rise. The pieces are fabricated by Trans-Tech and sent to SLAC for cleaning and plating. The parts are shipped to the IVU manufacturer, Neomax Engineering Co., for installation to the IVU.

Finite element modeling shows that copper plating the ferrite contact surface significantly reduces the temperature rise from the dissipated rf power. The contact pressure from the $1000 \mathrm{~N}$ clamp force is $8.8 \mathrm{MPa}$ with a thermal contact conductance of at least $\mathrm{hc}=3000 \mathrm{~W} / \mathrm{m}^{2}-^{\circ} \mathrm{C}$ over a heat transfer contact area of $113 \mathrm{~mm}^{2}$. Thermal power deposition is primarily in the high B field volumes near the split plane of the ferrite pair. The finite element model of the ferrite with a bare contact surface and $4 \mathrm{~W}$ dissipated shows a peak temperature rise of $267^{\circ} \mathrm{C}$. The addition of $100 \mu \mathrm{m}$ of $\mathrm{Cu}$ plating to the ferrite contact surface reduces the peak 
temperature rise to $100^{\circ} \mathrm{C}$. The contact surfaces are $\mathrm{Cu}$ plated using the SLAC cyanide copper process. A metallized strike coating is required prior to copper plating. The SLAC Klystron group is able to sputter coat Ti to provide this metallization. After assembly to the IVU, a thermocouple is clamped to two of the ferrite pieces to monitor the rf heating temperature rise.

The ferrites are vertically separated from the magnetic poles by $82 \mathrm{~mm}$. A two dimensional, quarter period model of the undulator including the ferrite, with a DC relative permeability $\mu_{r}=317$, confirms that the ferrite pieces have no significant effect on the undualtor field.

\section{TEST RESULTS AND FURTHER DISCUSSIONS}

In the new BL17 ID, our design of ferrite dampers are successfully incorporated; therefore, this device can serve as the first test-bed for the performance of these dampers. In this section, we will discuss the rf cold measurement results and compare them with the results for BL15ID.

\section{A. Cold rf tests of BL17 IVU with ferrite dampers}

Due to some technical constraints, only 22 pairs, instead of the intended 24 pairs of ferrite brackets, were eventually installed in the chamber. For the rf measurement, we duplicate the experimental setup that we had used for the rf measurement of the BL15 ID. As expected, the transmission measurement for $S_{21}$ clearly show the first three HOM modes. We vary the magnet gap of the ID from $6.785 \mathrm{~mm}$ to $10 \mathrm{~mm}$ at $0.2 \mathrm{~mm}$ per step and measure the center frequencies and loaded $Q \mathrm{~s}$ of the first three modes at each gap settings. The measured results are shown in Fig. 12 for both the resonant frequencies (top plot) and the loaded $Q$ s (bottom plot) of each of the three rf modes. Due to the nearly identical design, we expect the properties of the HOM modes in both BL17 ID and BL15 to be similar. In fact, the difference in resonant frequencies of mode 3 of the two devices is within 10\%. This discrepancy in frequency can be caused by many detailed factors in the IVU construction, such as subtle differences in structures and the addition of ferrite materials. The discrepancy can also partly be caused by the difference between published and actual pole gap. It is worth noting that it has been a challenging task to measure the exact visible clearance aperture in an IVU in SPEAR3 and verify that the actual gap size agrees with manufactures measured gap size. Although it will be beneficial to better understand the causes of this difference, the slight difference in these resonant frequencies is unimportant for the purpose of this study to damp the trapped mode.

The performance of the ferrite dampers is well illustrated in the bottom plot of Fig. 12, where the measured loaded $Q$ of BL17 ID at various gaps and that of BL15 ID (pentagrams) at $6.82 \mathrm{~mm}$ are shown together for comparison. The values for the loaded $Q$ of the first three modes for BL15 ID (a)

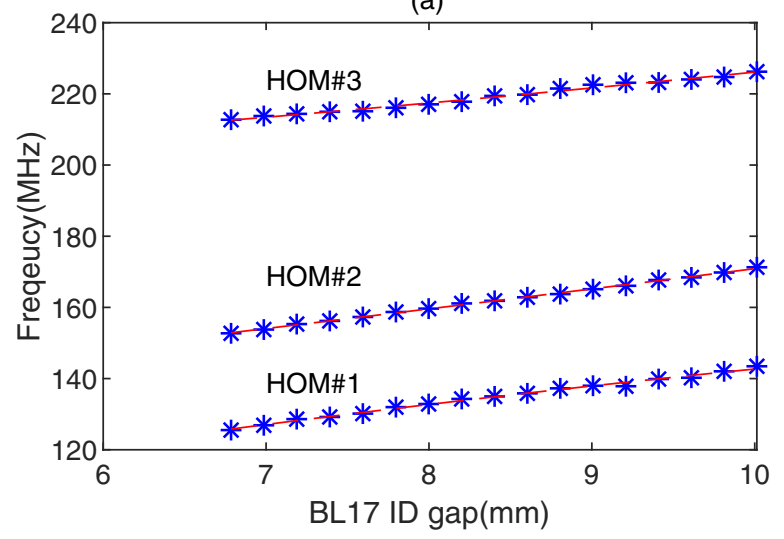

(b)

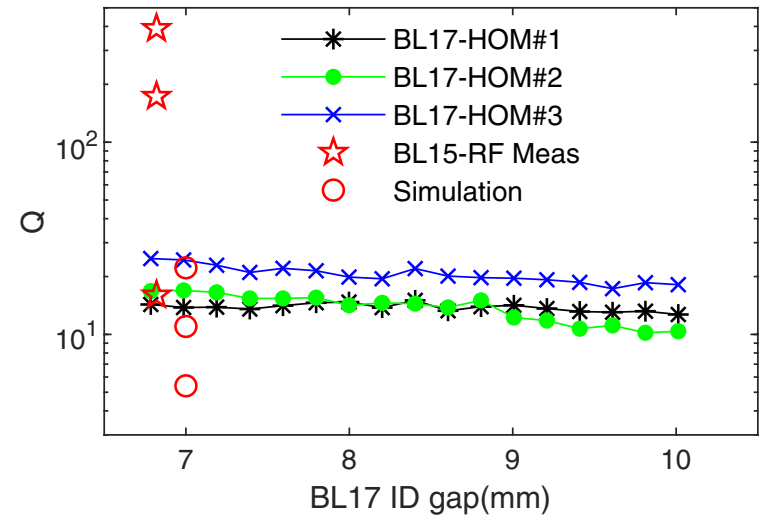

FIG. 12. rf cold test results for BL17 ID with ferrite dampers: rf modes frequencies vs ID magnet gap (top) and $\mathrm{rf}$ modes $Q$. vs ID magnet gap (bottom).

at $6.82 \mathrm{~mm}$ gap are 16,173, and 390 respectively. After adding the dampers in BL17 ID, at $6.79 \mathrm{~mm}$ gap, the loaded $Q$ of these three modes are $14.3,16.8$, and 24.8 , respectively. The modes are not only effective damped in order to prevent beam instabilities, the measurements also show good agreement with the simulation results, shown as red circles in the plot, which predict the $Q$ of HOM \#3 to be 20.2. However, the simulation predicts much stronger damping effects for HOM \#1 and \#2. These differences have no impact in solving the problem of beam instabilities caused by these trapped $\mathrm{rf}$ modes. The reasons for the differences are likely due to details included in the modeled structure.

\section{B. Discussions of other approaches}

Before converging to the solution of using ferrite dampers to suppress the HOMs in the IVU chamber, we had explored several other approaches. We investigated adding mode curtains to the structure, different transition geometries in the device, as well as, several other kinds of damping schemes. Though the ferrite dampers provide the most effective and simplest solution for us, we believe that it is worth summarizing our findings with two of these other 


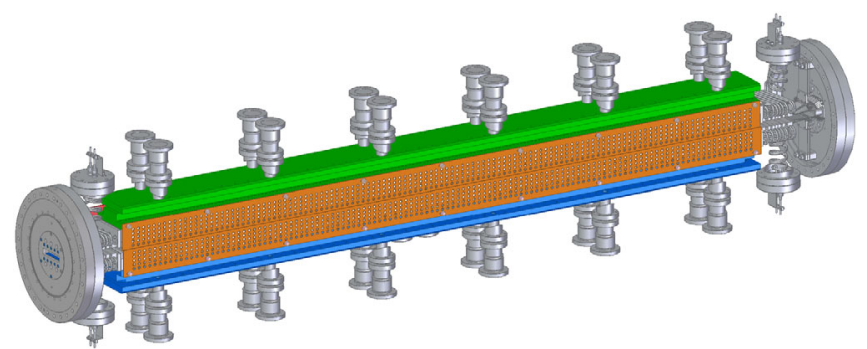

FIG. 13. Preliminary design of mode curtains for damping the trapped modes. (Courtesy of Neomax Engineering Co.)

approaches, namely mode curtains and a multi-turn loop antenna.

As shown in Fig. 13, we have completed a preliminary design of mode curtains. These are metal sheets attached to the magnet jaws. Their purpose is to electromagnetically shield the magnet gap from the rest of the chamber. Our idea of adding mode curtains to the IVU is inspired by the design of some IVUs in Diamond Light Source (DLS) [40]. Inside IVUs in DLS, overlapping metal perforated sheets are mounted on both sides of the magnet jaws. Sliding contacts on the transition tapers also are intended to contain the field. Though the design purpose of these additional structures in DLS is unclear, we believe that they can help in migrating the issues of low frequency mode trapping in the chamber.

As shown in Eq. (1), the coupling impedance of a mode is dependent on geometry factor $(R / Q)_{\perp}$ as well as the loaded quality factor $Q_{L}$. By adding ferrite dampers, we solve the HOM problem by reducing the value of $Q_{L}$, while largely keeping the basic IVU structure unchanged. With the mode curtains, the goal is to reduce the impedance by reducing $(R / Q)_{\perp}$. After some simulation studies with the mode curtains, we found that they are effective in substantially reducing the coupling impedance only when both the magnet jaws and the transition tapers are covered. This is as expected from the Panofsky-Wenzel Theorem [41], which indicates that, in a cavity structure, the transverse deflecting voltage to the beam from a resonant mode is derived from the transverse gradient of the integrated longitudinal electric field. In other word, if a particle beam travel through a resonant structure without experiencing any longitudinal electric field, the net effects on transverse deflection will be zero. In Fig. 14, we plot the longitudinal electric field calculated from simulations along the IVU structure for both on axis and off axis by $1 \mathrm{~mm}$. The particles experience longitudinal fields only at the transition tapers, therefore, the geometry feature of the transition taper is critical to the transverse coupling impedance, as we have indicated earlier in this paper. Ideally, the mode curtains for the magnet jaws and transition tapers should attach to the structure tightly without any space in between; however, in SPEAR3, we have to maintain a minimum distance of $40 \mathrm{~mm}$ between the mode curtains and the

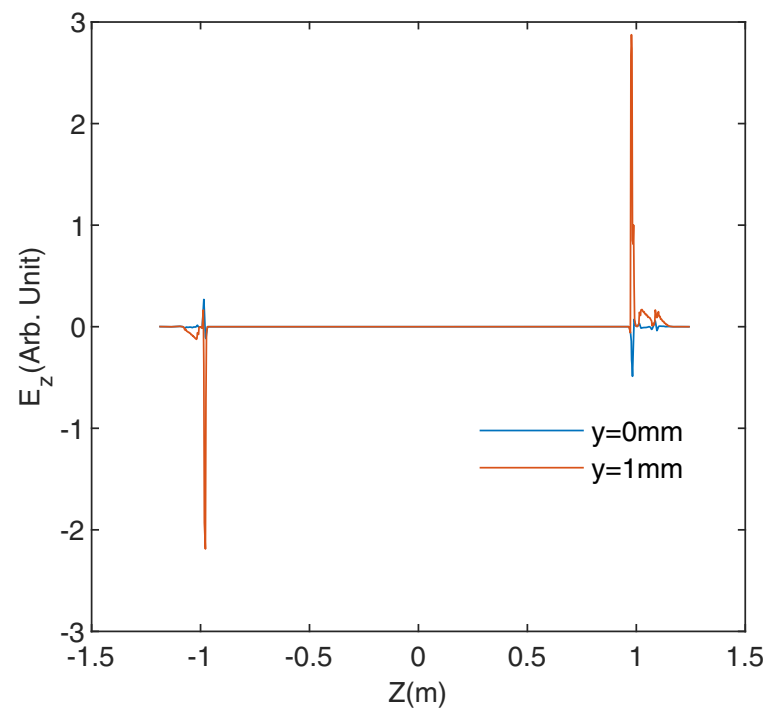

FIG. 14. On axis and off axis longitudinal electric fields for HOM \#3 along the IVU device.

outboard magnet jaw in order to avoid a synchrotron radiation strike on the curtain. Our numerical simulations show that, with such a large space between the curtains and the structure, the damping effects are significantly compromised. In Fig. 15, we show the simulation results of transverse coupling impedance for the TE modes up to about $1.7 \mathrm{GHz}$ in three cases: without any mode curtains (red circles), ideal curtains being attached right against the structure (green stars), and one side of the curtains displaced by $40 \mathrm{~mm}$ from the IVU magnet jaw and transition tapers (black triangles). In the case with ideal curtains, the mode frequencies are pushed up and the impedance for modes below $1 \mathrm{GHz}$ drop to nearly zero. However, after adding a $40 \mathrm{~mm}$ space between the curtains

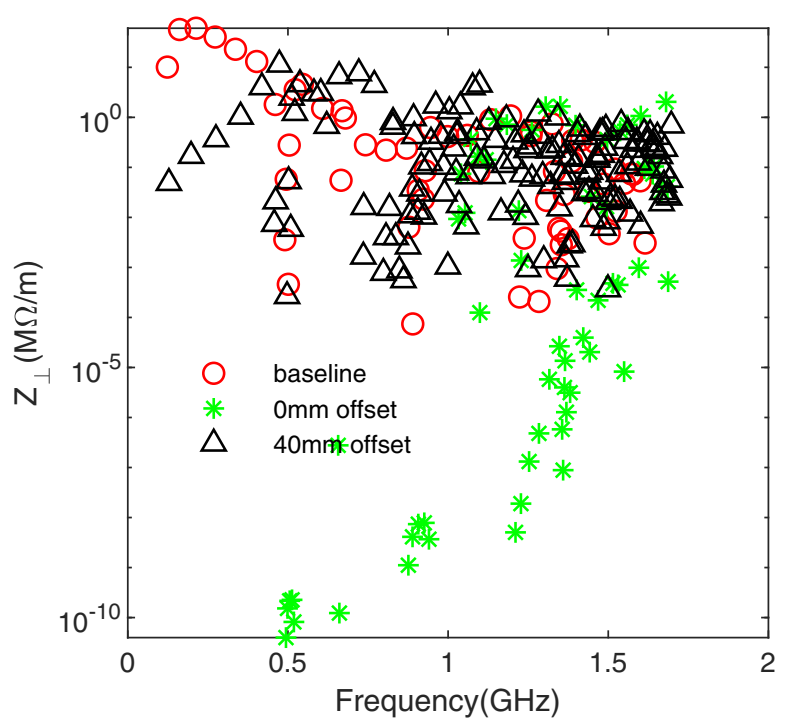

FIG. 15. Simulation results with mode curtains. 
and the IVU structure, the curtains have very little effect on the mode frequency. The impedances of the modes below $400 \mathrm{MHz}$ are damped significantly compared to the original IVU structure, but those of some higher frequency modes, e.g., some modes between $600 \mathrm{MHz}$ and $800 \mathrm{MHz}$, increase significantly. Since our greatest mismatch between simulations and measurements has been the value of $Q_{L}$, without more study, we cannot convince ourselves that the actual $Q_{L}$ will not be higher. Overall, our predicted effects of using the mode curtain will not be as straightforward as our prediction of using ferrite dampers. However, we believe that further studies should be conducted to evaluate this method, since we believe that it can be a viable solution for other facilities with fewer constraints on the location of the mode curtains with respect to the IVU magnet.

Beside the mode curtains, we also considered a multiturn loop antenna to couple the power out of the chamber so that the loaded $Q_{L}$ can be damped. Due to the long wavelength of the modes of concern, it is impossible to couple the modes out via waveguide. An antenna with a single loop can serve as a broad band coupler, but due to the limitation of port size and space, the largest loop we can put in still only has a free space radiation impedance of $0.3 \mathrm{ohm}$ at $200 \mathrm{MHz}$. Since multiturn loops increase the radiation impedance, we designed a 9-turn loop antenna to increase the coupling impedance. However, preliminary simulation results showed that the bandwidth of loop structure becomes narrow as the number of loops increases and cannot easily couple with the mode in the chamber. Based on these less promising simulation results, we abandoned the test plan for the multiturn loop antenna.

\section{CONCLUSION}

When, at SPEAR3, we observed coupled-bunch instabilities caused by one of our IVUs, we were motivated to conduct thorough studies on their sources. This led us to conduct some pioneering R\&D studies in understanding and controlling the beam coupling impedances caused by the trapped rf modes in the IVU chamber. With beam-based measurements, rf measurements, cold and with beam, and numerical simulations, we characterized the source of the beam instabilities in the IVU chamber. The clear understanding we obtained of the rf properties of the trapped rf modes enabled us to design an effective and simple solution using ferrite dampers to suppress the trapped modes and eliminate the instabilities. The results of the first cold rf measurement on BL17 ID in SPEAR3, the IVU equipped with these ferrite dampers, indicated that their damping effects agree with numerical simulations. Therefore, we will not expect this new IVU to introduce coupled-bunch instabilities even when operating passively without $\mathrm{BxB}$ feedback. Results of beam tests will be available in the near future during the ID commissioning for user operation.

In SPEAR3, we have developed an effective migration technique for the coupled-bunch instabilities caused by the IVU, nonetheless, more studies are required to fully understand the exact impedances effects from the IVU device. Such studies are beyond the operational requirement of SPEAR3, but are important for future diffraction limited storage ring facilities, for which, the impedance budgets are harder to meet due to small vacuum chambers. In order to explore the effects of the complex geometric features and material properties of the IVU device on the beam coupling impedances, extra efforts will be required to improve the numerical model for the simulations. Furthermore, it is desirable to directly measure the transverse coupling impedance of the IVU device using a beam based technique or on bench low level rf techniques so that the results can be compared with numerical simulations for benchmark. All these studies will be beneficial to both the next generation SR facilities for high brightness operation and the IVU designers to minimize the impedance effects from the design phase.

\section{ACKNOWLEDGMENTS}

We appreciate the useful discussions with J. Safranek, T. Rabedau, D. Teytelman, X. Huang, N. Kurita, V. Dolgashev, and A. Krasnykh. We would like to acknowledge J. Vargas, B. Evanson, P. Velikov, G. Lanza, T. Dinan, and A. Haase for their technical supports in testing and measurements. We want to thank G. Rehm, S. Milward, J. Chavanne, E. Wallen, S. Santis, and R. Dowd for sharing with us their operating experiences with IVUs. We also would like to thank Skyworks and Trans-Tech for supplying the ferrite pieces and the Neomax Engineering Co. for their helpful consultations and advice during the mode damper design and for assembling the ferrite pieces to the IVU. This work is supported by the U.S. Department of Energy under Contract No. DE-AC02-76SF00515. This research used resources of the National Energy Research Scientific Computing Center (NERSC), a U.S. Department of Energy Office of Science User Facility operated under Contract No. DE-AC02-05CH11231.

[1] S. Yamamoto, T. Shioya, M. Hara, H. Kitamura, X. W. Zhang, T. Mochizuki, H. Sugiyama, and M. Ando, Construction of an in-vacuum type undulator for production of undulator $\mathrm{x}$ rays in the $525 \mathrm{kev}$ region, Rev. Sci. Instrum. 63, 400 (1992).

[2] H. Kitamura, Insertion devices for third-generation light sources, Rev. Sci. Instrum. 66, 2007 (1995).

[3] J. Chavanne, C. Penel, B. Plan, and F. Revol, In-vacuum undulators at ESRF, in Proceedings of the 2003 Particle Accelerator Conference, Portland, OR, 2003 IEEE, New York, 2003), p. 253.

[4] J. Campmany, D. Bertwistle, J. Marcos, Z. Mart, V. Massana, F. Becheri, and D. Einfeld, A general view of IDs to be installed at alba on day one, in Proceedings of the 10th European Particle Accelerator Conference, 
Edinburgh, Scotland, 2006 (EPS-AG, Edinburgh, Scotland, 2006), p. 3598.

[5] G. Ingold, M. Boege, W. Bulgheroni, A. Keller, J. Krempaski, C. Schulze-Briese, L. Schulz, T. Schmidt, D. Zimoch, T. H. T. Tanaka, and H. Kitamura, Performance of small-gap undulators at the SLS intermediate energy storage ring, AIP Conf. Proc. 879, 388 (2007).

[6] E. C. Longhi, R. Bartolini, R. Fielder, I. Martin, J. C. Schouten, and B. Singh, First year's experience of DIAMOND insertion devices, in Proceedings of the 11th European Particle Accelerator Conference, Genoa, Italy, 2008 (EPS-AG, Genoa, Italy, 2008), p. 2285.

[7] J.-C. Huang, L.-H. Wu, C.-K. Yang, C.-K. Chuan, T.-Y. Chung, and C.-S. Hwang, Performance of a NSRRC invacuum undulator, IEEE Trans. Appl. Supercond. 24, 0503704 (2014).

[8] T. Tanabe, P. Cappadoro, T. Corwin, H. Fernandes, D. A. Harder, Y. Hidaka, C. Kitegi, M. Musardo, and J. Rank, Insertion devices at the national synchrotron light source-II, Synchrotron Radiat. News 28, 39 (2015).

[9] H. Kitamura, T. Hara, T. Tanaka, T. Bizen, X. Maréchal, and T. Seike, Design criteria and technology challenges for the undulators of the future, in Proceedings of the 9th European Particle Accelerator Conference, Lucerne, Switzerland, 2004 (EPS-AG, Lucerne, Switzerland, 2004), p. 59.

[10] J.-C. Huang, H. Kitamura, C.-K. Yang, C.-H. Chang, C.-H. Chang, and C.-S. Hwang, Challenges of in-vacuum and cryogenic permanent magnet undulator technologies, Phys. Rev. Accel. Beams 20, 064801 (2017).

[11] R. Reiser, S. Zelenika, G. Ingold, A. Keller, L. Schulz, T. Hara, and H. Kitamura, The flexible taper transitions for an in-vacuum undulator, in Proceedings of MEDSI'02, Chicago, USA, 2008 (Argonne National Laboratory, Argonne, Illinois USA, 2008), p. 323, https://medsi.lbl .gov/files/page_135/Papers/04_Insertion_Devices_and_ Vacuum_Chambers/The_Flexible_Taper_Transitions_for_ an_In-Vacuum_Undulator_-_R._Reiser_S._Zelenika_G._ Ingold_A._Keller_L._Schulz_T._Hara_H._Kitamura.pdf.

[12] A. Blednykh, S. Krinsky, B. Podobedov, and J.-M. Wang, Transverse impedance of small-gap undulators for NSLS-II, in Proceedings of the 10th European Particle Accelerator Conference, Edinburgh, Scotland, 2006 (EPSAG, Edinburgh, Scotland, 2006), p. 2970.

[13] A. Blednykh et al., First collective effects measurements in NSLS-II with IDs, in Proceedings of the 6th International Particle Accelerator Conference, Richmond, Virginia, USA, 2015 (JACoW, Richmond, Virginia, USA, 2015), p. 1332 .

[14] V. Smaluk, R. Fielder, A. Blednykh, G. Rehm, and R. Bartolini, Coupling impedance of an in-vacuum undulator: Measurement, simulation, and analytical estimation, Phys. Rev. ST Accel. Beams 17, 074402 (2014).

[15] R. Dowd, M. Atkinson, M. J. Boland, G. S. Leblanc, Y.-R. E. Tan, and D. Teytelman, Investigation of trapped resonant modes in insertion devices at the Australian Synchrotron, in Proceedings of the 7th International Particle Accelerator Conference, Busan, Korea, 2016 (JACow, Busan, Korea, 2016), p. 1710.
[16] K. Tian, J. Sebek, and J. L. Vargas, Investigation of transverse beam instability induced by an in-vacuum undulator at SPEAR3, in Proceedings of the 2016 International Beam Instrumentation Conference, Barcelona, Spain, 2016 (JACoW, Barcelona, Spain, 2016), p. 31.

[17] G. Rehm, Measurement of rf resonances and measured impact on transverse multibunch instabilities from invacuum insertions devices, in Proceedings of the 8th International Particle Accelerator Conference, Copenhagen, Denmark, 2017 (JACoW, Copenhagen, Denmark, 2017), p. 3188.

[18] R. Bartolini, R. Fielder, and G. Rehm, Multi-bunch instabilities measurement and analysis at the Diamond Light Source, in Proceedings of the 8th International Particle Accelerator Conference, Copenhagen, Denmark, 2017 (JACoW, Copenhagen, Denmark, 2017), p. 4485.

[19] S. B. Cohn, Properties of ridge wave guide, Proc. IRE 35, 783 (1947).

[20] S. Hopfer, The design of ridged waveguides, IRE Trans. Microwave Theory Tech. 3, 20 (1955).

[21] S. Ramo, J. R. Whinnery, and T. V. Duzer, Fields and Waves in Communication Electronics (John Wiley \& Sons, Inc., 1994).

[22] A. Chao, Physics of Collective Instabilities in High Energy Accelerators (John Wiley \& Sons, Inc., New York, 1993).

[23] D. Teytelman, Overview of system specifications for bunch by bunch feedback systems, in Proceedings of the 2011 Particle Accelerator Conference, New York, USA, 2011 (IEEE, New York, 2011), p. 1475.

[24] D. Teytelman, Optimization of bunch-to-bunch isolation in instability feedback systems, in Proceedings of the 2013 International Beam Instrumentation Conference, Oxford, UK, 2013 (JACoW, Oxford, UK, 2016), p. 116.

[25] M. Lonza, Multi-bunch feedback systems, in 2007 CERN Accelerator School (Sigtuna, Sweden, 2007), http://cds .cern.ch/record/1213287/files/p467.pdf.

[26] Y. Kang, G. Decker, and J. Song, Damping spurious harmonic resonances in the APS storage ring beam chamber, in Proceedings of the 1999 Particle Accelerator Conference, New York, USA, 1999 (IEEE, New York, 1999), p. 3092.

[27] G. Rehm, M. Abbott, and A. Morgan, New features and measurements using the upgraded transverse multibunch feedback at Diamond, in Proceedings of the 2014 International Beam Instrumentation Conference, Monterey, USA, 2014 (JACoW, Monterey, USA, 2014), p. 696.

[28] K. Harkay, T. Berenc, L. Emery, U. Wienands, D. Teytelman, J. Byrd, and R. Dowd, Characterizing the coupled bunch driving terms in a storage ring, in Proceedings of the 2017 International Beam Instrumentation Conference, Grand Rapids, USA, 2017 (JACoW, Grand Rapids, USA, 2017), p. 21.

[29] J. C. Slater, Microwave Electronics (D. Van Nostrand Company, Inc., Princeton, New Jersey, USA, 1950).

[30] D. M. Pozar, Microwave Engineering, 4th ed. (John Wiley \& Sons, Inc., New York, 2012).

[31] O. Kononenko, L. Ge, K. Ko, Z. Li, C.-K. Ng, and L. Xiao, Advances in massively parallel electromagnetic 
simulation suite ACE3P, in Proceedings of ICAP 2015 (Shanghai, China, 2015), p. 183, https://conf.slac.stanford .edu/cw18/.

[32] NERSC website: http://www.nersc.gov/.

[33] CUIBIT website: https://cubit.sandia.gov/.

[34] ParaView website: https://www.paraview.org/.

[35] N. Biancacci, F. Caspers, J. Kuczerowski, E. Métral, N. Mounet, B. Salvant, A. Mostacci, O. Frasciello, and M. Zobov, Impedance simulations and measurements on the LHC collimators with embedded beam position monitors, Phys. Rev. Accel. Beams 20, 011003 (2017).

[36] A. Bertarelliand and M. Garlaschè, Design guidelines for ferrite absorbers submitted to rf-induced heating, in Proceedings of the 4th International Particle Accelerator Conference, Shanghai, China, 2013 (JACow, Shanghai, China, 2013), p. 3394.

[37] TT2-111R Data Sheet, courtesy of Trans Tech, Adamstown, Maryland.
[38] G. Lanza, Outgassing test of Trans-Tech TT2-111R, Internal SLAC Report No. SSRL-Eng-Note M829, 2017.

[39] T. Tajima, K. Asano, T. Furuya, Y. Ishi, Y. Kijima, M. Kudo, S. Mitsunobu, T. Takahashi, and N. Taniyama, Development of HOM absorber for KEK B-Factory SC Cavities, in Proceedings of the 6th Workshop on RF Superconductivity, Newport Newport News, VA, USA (CEBAF, Newport News, VA, USA, 1993), p. 962, https://accelconf.web.cern.ch/accelconf/SRF93/papers/ srf93i39.pdf.

[40] G. Rehm (private communication).

[41] W. K. H. Panofsky and W. A. Wenzel, Some considerations concerning the transverse deflection of charged particles in radiofrequency fields, Rev. Sci. Instrum. 27, 967 (1956).

Correction: One of the names listed in the Acknowledgments section was misspelled and has been fixed. 